\title{
Acute toxicity profile in prostate cancer with conventional and hypofractionated treatment
}

\author{
Gustavo Arruda Viani ${ }^{1,3^{*}}$, Lucas Bernardes Godoy da Silva', Bruna Bueno da Silva', Yuri Bonicelli Crempe', \\ Vinicius Spazzapan Martins ${ }^{2}$, Ricardo Jose Rambaiolo Ferrari ${ }^{1}$, Mariana Colbachini Pólo ${ }^{2}$, Bruno Thiago Rossi ${ }^{1}$, \\ Elton Suguikawa ${ }^{1}$, Giseli Correa Zulliani ${ }^{1}$ and Eduardo Jose Stefano ${ }^{1}$
}

\begin{abstract}
Purpose: To compare the acute toxicities in radical treatment of prostate cancer between conventional schedule (C-ARM) with 78 Gy/39 fractions and hypofractionation conformal treatment (H-ARM) with 69 Gy/23 fractions.

Methods and material: This prospective double arm study consisted of 217 patients with prostate cancer, 112 in H-ARM and 105 in C-ARM arm. C-ARM received conventional six- field conformal radiotherapy with 78 Gy in 39 fractions while H-ARM received hypofractionation with 69 Gy in 23 fractions. Weekly assessment of acute reactions was done during treatment and with one, and 3 months using RTOG scale. Univariated analysis was performed to evaluate differences between the incidences of acute reaction in the treatment arms. Variables with $p$ value less than 0.1 were included in the multivariated logistic regression.

Results: There was no difference between H-ARM versus C-ARM for severity and incidence in genitourinary (GU) and gastrointestinal $(\mathrm{Gl})$ acute toxicity. During the treatment comparing H-ARM with C-ARM no differences was observed for $\mathrm{Gl}$ toxicity (grade $0-3 ; \mathrm{H}$-ARM $=45.5 \%, 34 \%, 18.7 \%$ and $1.8 \%$ versus C-ARM $=47.6 \%, 35.2 \%, 17.2 \%$ and 0 ). For acute GU toxicity no difference was detected between H-ARM (grade $0-3 ; 22.3 \%, 54.5 \%, 18.7 \%$ and $4.5 \%$ ) and C-ARM (grade $0-3 ; 25.8 \%, 53.3 \%, 17.1 \%$ and $3.8 \%$ ).

At the 3- months follow-up, persistent Grade > =2 acute GU and GI toxicity were 2.5\% and 1.8\% in H-ARM versus 5.7\% and $3 \%$ in C-ARM $(p>0.05)$. In univariated and multivariated analyses, there was not any dosimetric predictor for $\mathrm{Gl}$ and GU toxicity.
\end{abstract}

Conclusions: Our data demonstrate that hypofractionated radiotherapy achieving high biological effective dose using conformal radiotherapy is feasible for prostate cancer, being well tolerated with minimal severe acute toxicity.

Keywords: Acute toxicity, Prostate cancer, Hypofractionation, Conformal radiotherapy

\section{Introduction}

In the last decades, it has been shown that exists a dose-response relation between the prostate cancer biochemical control and the total dose of radiotherapy delivered [1]. Evidences have also been growing from experimental and clinical studies that the $\alpha / \beta$ ratio of the linear-quadratic formulation for prostate cancer might be between 1.5 and 1.85 Gy [2-4]. This low $\alpha / \beta$ ratio suggests that prostate cancer has high sensitivity to

\footnotetext{
* Correspondence: gusviani@gmail.com

${ }^{1}$ Department of Radiation Oncology, Marilia Medical School, Marília, São Paulo, Brazil

${ }^{3}$ Department of Radiation Oncology, Marilia Medical School, Maria Cecilia Alves, 192, Marilia, São Paulo, Brazil

Full list of author information is available at the end of the article
}

dose per fraction, which suggests that a hypofractionation, with a large radiation dose delivered in a smaller number of fractions, might be more advantageous when compared to other type of cancer cells. On the other hand, the $\alpha / \beta$ ratio of the rectum is as important as that of prostate cancer for exploring which hypofractionation regimens will be most beneficial. Although $\alpha / \beta$ ratio for the rectal wall is not known precisely, animal studies suggest $\alpha / \beta$ ratio for the rectum of 4-6 G [5] . If the $\alpha / \beta$ ratio for rectum is higher than that for prostate, theoretically, larger hypofractionated doses could be given with larger clinical gains within the same or lower complication rates [6].

Although the hypofractionation schedule for prostate cancer appears more attractive than conventional fractionation,

\section{Biomed Central}


the experience of using hypofractionation with total equivalent doses of 78-80 Gy has been limited.

These limited data are from a randomized Phase III trial comparing a conventional fractionation regimen of 80 Gy given in 2-Gy fractions with a hypofractionation regimen of 62 Gy given in 20 fractions of 3.1 Gy/d [7], a nonrandomized study of hypofractionation vs. conventional fractionation delivered using $3 \mathrm{D}$ conformal radiotherapy technique [8], and a few other Phase I-II reports using image guide radiotherapy (IGRT) or intensitymodulated radiotherapy (IMRT) [9-11].

In order to compare two-fractionation regimens of radiotherapy in prostate cancer patients, we compared a high dose hypofractionation schedule (69 Gy/ 23 fractions) with conventional fractionation (78 Gy/39 fractions). This report summarizes the acute genitourinary and gastrointestinal side effects for all patients included in our prospective nonrandomized study, comparing conventional or hypofractionated RT.

\section{Material and methods}

It is a prospective study conducted after the approval of the institutional review board. The study population consisted of 217 patients with localized prostate cancer, who were treated between November 2009 and January 2011, with patients selected into two arms. Patients in C-Arm received conventional radiotherapy and those in $\mathrm{H}$-Arm received hypofractionated radiotherapy, both treatments with conformal technique.

\section{Evaluation}

The pretreatment evaluation consisted of a full history, with special emphasis on pretreatment urinary and rectal symptoms, and a physical examination. The prognostic groups were defined as follows: low risk, Stage T1-T2a, Gleason score $<7$, and initial prostate-specific antigen (iPSA) level $<10 \mathrm{ng} / \mathrm{mL}$; intermediate risk, Stage T1-T2b, Gleason score $<7$, and iPSA level of $10-19.9 \mathrm{ng} / \mathrm{mL}$ or Stage T1-T2b, Gleason score 7, and iPSA <20 ng/mL; and high risk, Stage T3, Gleason score 8-10, or iPSA > 20 ng/mL. Patients with metastases were excluded this trial.

\section{Selection for treatment arms}

Patients were selected for treatment arms according to their convenience. This bias was permitted, because the most patients came from long distances to treat. The groups were balance to achieve similar distribution between the treatment arms. If a patient was chosen to be treated in the $\mathrm{H}$ - Arm, the next patient automatically was allocated in $\mathrm{C}$-arm.

\section{Treatment}

For each patient, a conformal radiotherapy plan consisting of six 6-MV photon beams was used to deliver 69 Gy in
23 daily fractions of 3 Gy or 78 Gy in 39 fractions of 2 Gy, prescribed at the isocenter. No patient was treated with Intesity Modulated Radiotherapy (IMRT) and without any fiducial. This dose was used because, according to the linear quadratic formula, it is biologically equivalent to $87.8 \mathrm{~Gy}$ in $2 \mathrm{~Gy}$ fractions or $90 \mathrm{~Gy}$ in 50 fractions assuming an $\alpha / \beta$ ratio of 1.8 and $1.5 \mathrm{~Gy}$, respectively. All patients underwent a treatment planning CT (5-mm slice thickness) in the supine position with a triangle sponge placed under their knees. Patients were advised to have a comfortably full bladder and an empty rectum at time of computed tomography (CT) simulation. The prostate, seminal vesicles base, whole rectum, bladder, femoral heads, and penile bulb were contoured in all patients. The clinical target volume was the prostate plus seminal vesicles base; no patient had the pelvis included in the clinical volume. The planning target volume consisted of the clinical target volume plus a uniform $10-\mathrm{mm}$ margin in all directions, excluding the rectal margin $(7 \mathrm{~mm})$. All fields were treated daily. Single exposure portal films were obtained previous to the first fraction and weekly thereafter. Dose-volume histograms (DVHs) were calculated for a rectal volume encompassing the organ from anal merge to the retosigmoid transition. Rectal DVH constraints were V60 Gy $<=50 \%$, V65 Gy $<=35 \%$, V 70Gy $<=25 \%$ and $\mathrm{V} 75 \mathrm{~Gy}<=15 \%$ for C-Arm. In the H-Arm, DVHs were calculated from the standard 2-Gy fractionation equivalent to the doses of 3 Gy by fraction (assuming $\alpha / \beta=3 \mathrm{~Gy}$ ). Table 1 describes the rectal and Bladder DVH constrains used in H-Arm and C-Arm. The software used was Eclipse version 8.6, Varian Medical System, Inc, Palo Alto, USA. Patients considered as intermediate or high risk were treated with neoadjuvant, comcomitant and adjuvant androgen blockage. The intermediate risk received 6 months and high risk 24 months of androgen blockage. The androgen blockage consisted of a

\section{Table 1 Dose constrains}

\begin{tabular}{|c|c|c|}
\hline Variables & C-Arm & H-Arm \\
\hline \multirow[t]{2}{*}{ PTV } & $\begin{array}{l}95 \% \text { of } \mathrm{PTV} 78 \text { to } \\
\text { receive } 78 \mathrm{~Gy}\end{array}$ & $\begin{array}{l}95 \% \text { of PTV69 to } \\
\text { receive } 69 \mathrm{~Gy}\end{array}$ \\
\hline & (by definition) & (by definition) \\
\hline \multirow[t]{4}{*}{ Rectum } & V60 Gy $<=50 \%$ & V50Gy $<=50 \%$ \\
\hline & V65 Gy $<=35 \%$ & V54Gy $<=35 \%$ \\
\hline & V70Gy $<=25 \%$ & V58Gy $<=25 \%$ \\
\hline & V75Gy $<=15 \%$ & V62Gy $<=15 \%$ \\
\hline \multirow[t]{4}{*}{ Bladder } & V65Gy< $<50 \%$ & V54Gy< $<50 \%$ \\
\hline & V70Gy< $<=35 \%$ & V58Gy $<=35 \%$ \\
\hline & V75Gy $<=25 \%$ & V62Gy< $<25 \%$ \\
\hline & V80Gy< = 15\% & V67Gy< = 15\% \\
\hline Femurs & Maximal dose $<=55$ Gy & Maximal dose $<=46$ Gy \\
\hline
\end{tabular}


mensal intramuscular injection of acetate of goserelin of $3.6 \mathrm{mg}$.

\section{End points}

The primary study outcome was acute treatment reactions from the beginning of treatment to 3 months after the end of treatment. Patients were seen weekly, or as required, during treatment by a radiation oncologist. Preexistent urinary or rectal disorders, such as dysuria, pollakiuria, stress incontinence, hemorrhoids, and so forth, were assigned a grade complication if RT had exacerbated the baseline dysfunction. Acute gastrointestinal (GI) (retite, diarrhea, tenesmus and fecal incontinence) and genitourinary (GU) toxicity (dysuria, urinary frequency, retention, hematuria and urinary incontinence) were prospectively assessed and graded according to the Radiation Therapy Oncology Group scoring system for the rectum and bladder.

\section{Statistical analysis}

The association between the two groups was determined through bivariate analysis using Pearson's chi square test or Fisher test, when necessary. To compare continuous variable the student $T$ test was used. Two sided $p$ value was calculated and any difference with a $\mathrm{p}$ value $<0.05$ was considered significant. The dosimetric parameters such as; rectal, PTV and bladder volume were extracted from the treatment plan as well as age and ADT. These variables were tested whether they were related to the probability of having $>=$ Grade 2 RTOG toxicity. Univariated analysis and multivariated logistic regression were performed to evaluate differences between the incidences of acute reaction in the treatment arms. All statistical analysis was performed using SPSS (Statistical Analysis Systems software), version 19.

\section{Results}

A total of 217 patients (112 H-Arm and 105 C-Arm) treated on protocol from December 2009 to January 2011 were prospectively analyzed in this study. Baseline characteristics for all patients are shown in Table 2. The median age at last follow-up was 72 and 71 years for $\mathrm{C}$ Arm and $\mathrm{H}$-Arm, respectively. The follow-up for all patients was 3 months. Acute toxicity was weakly assessed during treatment and as needed by the patient before the regular follow-up. The majority of the patients tolerated the treatment well without major acute GI or GU side effects during the treatment $(47.6 \%$ in C-Arm and $45.5 \%$ in $\mathrm{H}$-arm for GI toxity and $25.8 \%$ in C-Arm versus $22.3 \% \mathrm{H}$-Arm for $\mathrm{GU}$ toxicity), as described in Table 3. The maximum acute GI and GU toxicities are shown for the whole group and for each fractionation schedule in Table 3. There was no apparent difference in any acute toxicity when compared the H-Arm to the
Table 2 Patients characteristics

\begin{tabular}{llll}
\hline Characteristic & C-Arm & Hypo-Arm & P value \\
\hline Patients (n) & 105 & 112 & \\
Age (median) & 72 & 71 & 0.913 \\
Baseline Gleason score & & & \\
$>7$ & 40 & 42 & 0.384 \\
$<=7$ & 65 & 70 & 0.765 \\
\hline Initial PSA level $\mathbf{( n g / m L )}$ & & & \\
Mean & 8.6 & 9.2 & 0.682 \\
Range & $0.72-58.4$ & $0.59-62.8$ & \\
\hline Risk stratification & & &
\end{tabular}

Risk stratification

$\begin{array}{llll}\text { Low risk } & 38 & 35 & 0.424\end{array}$

$\begin{array}{llll}\text { Intermediate Risk } & 44 & 40 & 0.485\end{array}$

$\begin{array}{llll}\text { High Risk } & 40 & 30 & 0.827\end{array}$

Androgen treatment

Follow-up (mo)

$84 \quad 70 \quad 0.938$

$\begin{array}{llll}\text { Mean rectal volume }(\mathbf{c m} 3) & 60.8+-2.9 & 59.7+-2.7 & 0.512\end{array}$

$\begin{array}{llll}\text { Mean bladder volume }(\mathbf{c m} 3) & 262.0+-16.5 & 258.9+-17.7 & 0.239\end{array}$

PTV Mean total volume (cm3) $\quad 167.5+-30.5 \quad 162.8+-31.2 \quad 0.114$

C-Arm ( $p>0.05$ ). During the treatment there were $23 \%$ of those in H-Arm and 19\% in C - Arm taking medication to improve urinary function (e.g., alpha blocker, antispasmodic, analgesic such as Pyridium). At 3 months of follow up, only 2 patients in $\mathrm{H}$-Arm (1.8\%) experiencing an acute GI toxicity score of 2 or more. Otherwise, in C Arm 4 patients (3.8\%) experienced an acute GI toxicity score of 2 , and 2 patients (1.9\%) experienced an acute GU toxicity score of 3 . Three patients in each arm developed acute GU toxicity Grade 3 (2.5\% in $\mathrm{H}$-arm and 3\% in Carm), as seen in Table 4. With 3-month follow up there were $15 \%$ and $13 \%$ in $\mathrm{H}$-Arms and C-Arms taking medication to improve urinary function; the difference was not significant.

Table 3 Incidence of maximum acute RTOG toxicity during the treatment

\begin{tabular}{llll}
\hline Characteristic & C-Arm & Hypo-Arm & P value \\
\hline GI toxicity & & & \\
Grade 0 & $50(47.6)$ & $51(45.5)$ & 0.643 \\
Grade 1 & $37(35.2)$ & $38(34)$ & 0.521 \\
Grade 2 & $18(17.2)$ & $21(18.7)$ & 0.235 \\
Grade 3 & 0 & $2(1.8)$ & 0.573 \\
\hline GU toxicity & & & \\
Grade 0 & $27(25.8)$ & $25(22.3)$ & 0.285 \\
Grade 1 & $56(53.3)$ & $61(54.5)$ & 0.594 \\
Grade 2 & $18(17.1)$ & $21(18.7)$ & 0.631 \\
Grade 3 & $4(3.8)$ & $5(4.5)$ & 0.432 \\
\hline
\end{tabular}


Table 4 Maximum acute RTOG toxicity at 3 months of follow up

\begin{tabular}{llll}
\hline Characteristic & $\begin{array}{l}\text { C-Arm (\%) } \\
\text { 105 patients }\end{array}$ & $\begin{array}{l}\text { Hypo-Arm (\%) } \\
\mathbf{1 1 2} \text { patients }\end{array}$ & P value \\
\hline Gl toxicity & & & \\
Grade 0 & $86(82)$ & $94(84)$ & 0.945 \\
Grade 1 & $13(12.3)$ & $16(14.2)$ & 0.221 \\
Grade 2 & $4(3.8)$ & $1(0.9)$ & 0.578 \\
Grade 3 & $2(1.9)$ & $1(0.9)$ & 0.683 \\
\hline GU toxicity & & & \\
Grade 0 & $81(77.1)$ & $84(75)$ & 0.321 \\
Grade 1 & $17(16.1)$ & $21(19)$ & 0.465 \\
Grade 2 & $4(3.8)$ & $4(3.5)$ & 0.987 \\
Grade 3 & $3(3)$ & $3(2.5)$ & 0.980 \\
\hline
\end{tabular}

No significant correlation was found among the dosimetric and clinical parameters such as; PTV volume, rectal volume, Bladder volume, age and androgen deprivation with acute GI and GU toxicity after 3 months of follow up, as described in Table 5. In the multivariate logistic regression no variable was associated with acute $\mathrm{GI}$ or GU acute toxicity, as described in Table 6.

\section{Discussion}

The present trial is the first nonrandomized study to compare a high-dose hypofractionated with conventionally fractionated schedule using conformal radiotherapy

Table 5 Maximum acute toxicity during follow up and clinical parameters

\begin{tabular}{|c|c|c|c|}
\hline \multirow[t]{2}{*}{ Characteristic } & \multicolumn{2}{|c|}{ Grade $>=2$ Acute Gl toxicity } & \multirow[t]{2}{*}{$P$ value } \\
\hline & C-Arm (\%) & H-Arm (\%) & \\
\hline ADT & $8(7.6)$ & $13(11.6)$ & 0.103 \\
\hline No ADT & $10(9.5)$ & $10(8.9)$ & \\
\hline PTV volume $>=165$ & $11(10.4)$ & $11(9.8)$ & 0.234 \\
\hline PTV volume $<165$ & $7(6.6)$ & $9(8)$ & \\
\hline Rectal volume $>=60$ & $9(8.5)$ & $11(9.8)$ & 0.545 \\
\hline Rectal volume $<60$ & $9(8.5)$ & $12(10.7)$ & \\
\hline Age $>=70$ & $11(10.4)$ & $12(10.7)$ & 0.756 \\
\hline \multirow[t]{2}{*}{ Age $<70$} & $8(7.6)$ & $8(7.1)$ & \\
\hline & \multicolumn{2}{|c|}{ Grade $>=2$ acute GU toxicity } & \\
\hline ADT & $10(9.5)$ & $12(10.7)$ & 0.645 \\
\hline No ADT & $12(11.4)$ & $14(12.5)$ & \\
\hline PTV volume $>=165$ & $13(12.3)$ & $15(13.3)$ & 0.453 \\
\hline PTV volume < 165 & $9(8.5)$ & $12(10.7)$ & \\
\hline Bladder volume $>=260$ & $9(8.5)$ & $13(11.6)$ & 0.987 \\
\hline Bladder volume < 260 & $13(12.3)$ & $14(12.5)$ & \\
\hline Age $>=70$ & $11(10.4)$ & $14(12.5)$ & 0.876 \\
\hline Age $<70$ & $11(10.4)$ & $13(11.6)$ & \\
\hline
\end{tabular}

Table 6 The multivariate analysis (logistic regression) evaluating the gastrointestinal or the genitourinary toxicity $>=$ grade 2

\begin{tabular}{llll}
\hline Gastrointestinal toxicity & & & \\
\hline Factor & Relative Risk & $\mathbf{9 5 \%} \mathbf{C l}$ & $\boldsymbol{P}$ \\
\hline Hypofractionation treatment & 1.03 & $0.45-15.56$ & 0.953 \\
No ADT & 0.87 & $0.27-15.72$ & 0.956 \\
Age $>=70$ years & 1.29 & $0.68-13.42$ & 0.765 \\
PTV volume $>=165$ & 1.33 & $0.72-11.14$ & 0.674 \\
Rectal volume $>=60$ & 0.98 & $0.38-7.55$ & 0.998 \\
\hline Genitourinary toxicity & & & \\
Hypofractionation treatment & 1.17 & $0.58-6.4$ & 0.978 \\
No ADT & 1.09 & $0.52-15.65$ & 0.743 \\
Age $>=70$ years & 1.13 & $0.59-14.01$ & 0.661 \\
PTV volume $>=165$ & 1.46 & $0.76-10.92$ & 0.932 \\
Bladder volume $>=260$ & 0.77 & $0.18-6.92$ & 0.839 \\
\hline
\end{tabular}

for prostate cancer. This study was designed to test the hypothesis that a high- dose hypofractionation regimen is equivalent to a conventional fractionation scheme in terms of acute GI and GU toxicity. This hypothesis was determined from the assumption that the $\alpha / \beta$ ratio would be 1.5-2 Gy for prostate cancer [3,4] and 10 and 3 Gy for early and slowly proliferating normal tissue, respectively. With the delivery of the same equivalent total dose to prostate tumors using a hypofractionation regimen, the corresponding equivalent doses to normal tissue would be lower. Therefore, with a slight prolongation of the shorter overall treatment time (from 4.5 weeks), both acute and late toxicity would be reduced compared with that occurring after conventional fractionation.

Based on this premise, this radiation protocol has been used at our institute since 2009. It was also empirically designed to provide acceptable biochemical control with satisfactory levels of toxicity, being convenient for the patients and with advantages to the hospital in terms of time and resource management. To date, the dose used in this trial is one of the highest BED delivered in a hypofractionated external beam regimen, as demonstrated in Table 7 [9,10,12-15]. Our results demonstrate that 69 Gy delivered in 23 daily fractions over 4.5 weeks is well tolerated using our RT technique and dose constraints, as seen in Table 7.

Moreover, the hypofractination schedule was quite well tolerated with more than $45 \%$ of patients presenting no acute GI toxicity during the treatment and at 3 months of follow up, only $2 \%$ of patients had residual grade $>=2$ toxicity. This data are comparable to other hypofractionation cohorts with residual Grade > =2 GI toxicity rates of $4-5 \%$. The incidence of Grade 2 or greater acute reactions reported in the present report for 
Table 7 Overview of published clinical data on hypofractionation in prostate cancer

\begin{tabular}{|c|c|c|c|c|c|c|c|c|c|}
\hline \multirow[t]{2}{*}{ References } & \multirow[t]{2}{*}{ Fractions } & \multirow[t]{2}{*}{ Fraction size } & \multirow[t]{2}{*}{ Total dose } & \multirow[t]{2}{*}{ weeks } & \multicolumn{3}{|c|}{ NTD2 for $\alpha / \beta$} & \multicolumn{2}{|c|}{ Acute toxicity } \\
\hline & & & & & $\alpha / \beta=1.5$ & $\alpha / \beta=3$ & $\alpha / \beta=10$ & GI (\%) & GU (\%) \\
\hline Livsey et al. [12] & 6 & 6 & 36 & 3 & 77.1 & 64.8 & 48 & & \\
\hline Kupelian et al. [9] & 28 & 2.5 & 70 & 5.5 & 80 & 77 & 72.9 & 19 & 15 \\
\hline Soete et al. [15] & 16 & 3.5 & 56 & 4 & 80 & 72.8 & 63 & 38 & 39 \\
\hline Madsen et al. [10] & 5 & 6.7 & 33.5 & 1 & 78.5 & 65 & 46.6 & 22 & 13 \\
\hline Pollack et al. [13] & 26 & 2.7 & 70.2 & 5 & 84.2 & 80.5 & 74.3 & 18 & 48 \\
\hline Martin et al. [14] & 20 & 3 & 60 & 4 & 77.1 & 72 & 65 & 12 & 25 \\
\hline FAMEMA trial (present study) & 23 & 3 & 69 & 4.5 & 88.7 & 83 & 76 & $20.5 \%$ & $23.2 \%$ \\
\hline
\end{tabular}

Abbreviations: $\mathrm{Gl}$ = gastrointestinal;GU = genitourinary; NTD2 = Equivalent dose (Gy) for 2-Gy fractionation; BED = biological effective dose.

our standard fractionation was not greater (rectal $17.2 \%$, urinary 20.9\%), than other trials of dose escalation. Acute toxicity has been addressed in several randomized clinical trials of dose escalation using standard fractionation. In a French trial [16], 30\% of patients presented with acute rectal reactions of Grade 2 or greater and $37 \%$ with urinary reactions of Grade 2 or greater in the arm treated to a mean dose of $78.5 \mathrm{~Gy}$. In another study from The Netherlands [17], gastrointestinal complications of Grade 2 or greater were experienced by $51 \%$ of patients in the 78-Gy arm and urinary complications of Grade 2 or greater in 55\%. Therefore, the use of five fractions weekly, instead of three or four, with an overall treatment time of 38 days for our hypofractionation regimen, have not increased the acute toxicity.

Another point that deserves attention is our margin given to the planning target volume (PTV). Despite the use of a larger posterior margin $(7 \mathrm{~mm})$ given to the PTV in our cohort, the rates of acute GI toxicity were similar to Soete et al. [15] delivering 56 Gy in 16 fractions of 3.5 Gy with $3 \mathrm{~mm}$ of margin. In their study was reported $5 \%$ of Grade 2 acute GI toxicity with no Grade 3 toxicity. For us, this satisfactory level of acute toxicity observed in our study can be result of an accurate treatment planning and set up verification, associated with a close attention to the dose-volume constraints for the organs at risk (Table 1). Although, does not exist a consensus on the optimal dosimetric parameters to be used in clinical practice. Rectal and bladder constraints are used to reduce the incidence of bladder and rectal toxicity. So, we speculate that although the dose-volume histograms based on the initial planning $\mathrm{CT}$ may not reflect the real dose received by the rectum because of displacement of the prostate and rectum during and between treatments, the use of restrictive DVH can help to maintain the acute toxicity rates in a satisfactory level.

In our univariated and multivariated analysis comparing dosimetric and clinical variables (e.g., risk group designation, PTV volume, SV irradiation, or hormonal therapy) between the two groups, no significant relationship with acute GI or GU effects was observed. This can be probably related to the large variability of bladder and rectum volumes during and between the treatments. This founds are in agreement with those of Pollack et al. [13], who also did not observe a target volume or normal tissue dose-volume dosimetric relationship with either acute GI or GU toxicity, nor with group designation or use of hormonal therapy.

\section{Conclusion}

We present here our early outcomes for a high dose hypofractionated conformal radiotherapy regime for the treatment of prostate cancer. This study suggests that hypofractionated dose-escalated radiotherapy using conformal radiotherapy in prostate cancer is feasible and produces acceptable toxicity with the dose constraints used. No difference in incidence or severity of acute gastrointestinal and genitourinary toxicity was observed when compared to conventional fractionation. Moreover, the acute toxicity rates were comparable to those of other dose escalation trials using standard or hypofractionated schedules, being transient, with only 2 patients having persistent Grade 2 or higher GI toxicity by 3 months follow up.

\section{Competing interests}

The author \& co-authors have no conflicts of interest to declare.

\section{Authors' contributions}

VGA, DsLBG and SEJ performed the statistical analysis and wrote the paper. DsBB, CYB, MVS, FRJR, PMC, RBT, ZGC and ES collected the data. All authors read and approved the final manuscript.

\section{Author details}

'Department of Radiation Oncology, Marilia Medical School, Marília, São Paulo, Brazil. ${ }^{2}$ Marilia Medical School, Marília (FAMEMA), São Paulo, Brazil. ${ }^{3}$ Department of Radiation Oncology, Marilia Medical School, Maria Cecilia Alves, 192, Marilia, São Paulo, Brazil.

Received: 9 January 2012 Accepted: 17 April 2013 Published: 21 April 2013

\section{References}

1. Viani GA, Stefano EJ, Afonso SL: Higher-than-conventional radiation doses in localized prostate cancer treatment: a meta-analysis of randomized, controlled trials. Int J Radiat Oncol Biol Phys 2009, 74:1405-1418.

2. Fowler JF: The radiobiology of prostate cancer including new aspects of fractionated radiotherapy. Acta Oncol 2005, 44:265-276. 
3. Fowler JF, Ritter MA, Fenwick JD, et al: How low is the alpha/beta ratio for prostate cancer? In regard to Wang et al., IJROBP 2003;55:194-203. Int J Radiat Oncol Biol Phys 2003, 57:593-595. author reply 595-596.

4. King CR, Fowler JF: A simple analytic derivation suggests that prostate cancer alpha/beta ratio is low. Int J Radiat Oncol Biol Phys 2001, 51:213-214.

5. Brenner $D$, Armour E, Corry P, et al: Sublethal damage repair times for a late-responding tissue relevant to brachytherapy (and external-beam radiotherapy): implications for new brachytherapy protocols. Int I Radiat Oncol Biol Phys 1998, 41:135-138.

6. Brenner DJ, Hall EJ: Fractionation and protraction for radiotherapy of prostate carcinoma. Int J Radiat Oncol Biol Phys 1999, 43:1095-1101.

7. Arcangeli G, Fowler J, Gomellini S, et al: Acute and late toxicity in a randomized trial of conventional versus hypofractionated threedimensional conformal radiotherapy for prostate cancer. Int J Radiat Oncol Biol Phys 2011, 79:1013-1021.

8. Leborgne F, Fowler J, Leborgne JH, et al: Later Outcomes and Alpha/Beta Estimate From Hypofractionated Conformal Three-Dimensional Radiotherapy Versus Standard Fractionation for Localized Prostate Cancer. Int J Radiat Oncol Biol Phys 2011, 82(3):1200-1207.

9. Kupelian PA, Willoughby TR, Reddy CA, et al: Hypofractionated intensitymodulated radiotherapy (70 Gy at 2.5 Gy per fraction) for localized prostate cancer: Cleveland Clinic experience. Int J Radiat Oncol Biol Phys 2007, 68:1424-1430.

10. Madsen BL, Hsi RA, Pham HT, et al: Stereotactic hypofractionated accurate radiotherapy of the prostate (SHARP), 33.5 Gy in five fractions for localized disease: first clinical trial results. Int J Radiat Oncol Biol Phys 2007, 67:1099-1105.

11. Coote JH, Wylie JP, Cowan RA, et al: Hypofractionated intensitymodulated radiotherapy for carcinoma of the prostate: analysis of toxicity. Int J Radiat Oncol Biol Phys 2009, 74:1121-1127.

12. Livsey JE, Cowan RA, Wylie JP, et al: Hypofractionated conformal radiotherapy in carcinoma of the prostate: five-year outcome analysis. Int J Radiat Oncol Biol Phys 2003, 57:1254-1259.

13. Pollack A, Hanlon AL, Horwitz EM, et al: Dosimetry and preliminary acute toxicity in the first 100 men treated for prostate cancer on a randomized hypofractionation dose escalation trial. Int J Radiat Oncol Biol Phys 2006, 64:518-526.

14. Martin JM, Rosewall T, Bayley A, et al: Phase II trial of hypofractionated image-guided intensity-modulated radiotherapy for localized prostate adenocarcinoma. Int J Radiat Oncol Biol Phys 2007, 69:1084-1089.

15. Soete G, Arcangeli S, De Meerleer $G$, et al: Phase II study of a four-week hypofractionated external beam radiotherapy regimen for prostate cancer: report on acute toxicity. Radiother Oncol 2006, 80:78-81.

16. Beckendorf V, Guerif S, Le Prise E, et al: The GETUG 70 Gy vs. 80 Gy randomized trial for localized prostate cancer: feasibility and acute toxicity. Int J Radiat Oncol Biol Phys 2004, 60:1056-1065.

17. Peeters ST, Heemsbergen WD, van Putten WL, et al: Acute and late complications after radiotherapy for prostate cancer: results of a multicenter randomized trial comparing $68 \mathrm{~Gy}$ to $78 \mathrm{~Gy}$. Int J Radiat Oncol Biol Phys 2005, 61:1019-1034.

\section{Submit your next manuscript to BioMed Central and take full advantage of:}

- Convenient online submission

- Thorough peer review

- No space constraints or color figure charges

- Immediate publication on acceptance

- Inclusion in PubMed, CAS, Scopus and Google Scholar

- Research which is freely available for redistribution 